\title{
Aim for the Sky, But Keep Your Feet on the Ground
}

\author{
To fight poverty and promote equality, developing countries should \\ pursue the highest levels of unfettered, open-ended scientific inquiry. \\ By Nithaya Chetty
}

deas around the development of African countries have progressed significantly since the 1960 s. In the initial stages

of postcolonial Africa, development support was often handed out to dictators who channeled those resources into their own bank accounts. Today, more democratic governments have shifted their priorities to strengthening education, advancing scientific research, fostering innovation, and developing talent.

Yet the advancement of African societies is painfully slow. Science and technology can be powerful catalysts for change, but the dearth of qualified scientists and the low levels of research funding pose formidable barriers to overcome. To

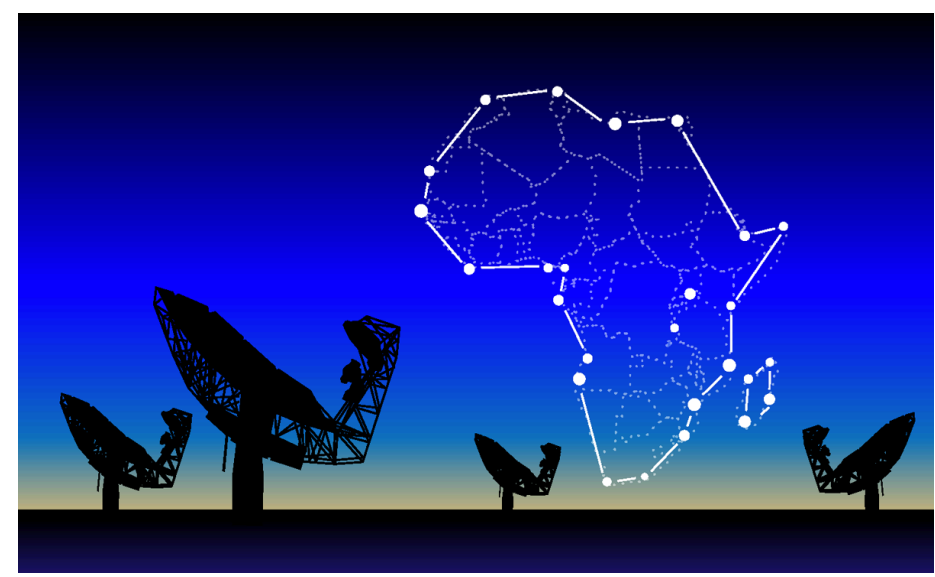

Artistic rendition of the MeerKAT radio telescope in South Africa's Northern Cape. The instrument is addressing fundamental astrophysics questions but also sparking commercial innovation and attracting many young students to science and technology. Credit: APS/Alan Stonebraker lower those barriers, African governments should support fundamental research without compromising on its standards. In doing so, they will build an environment that will strengthen economic development and mitigate the mass migration of people to foreign shores.

An all-too-common view-held by international institutions and by government agencies in Africa and elsewhere-is that for the foreseeable future, African countries should focus their efforts on education, rather than on research, by improving the mathematical literacy and other practical scientific skills of high-schoolers and undergraduates. The argument is that if we get the fundamentals right, we will eventually produce a critical mass of quality graduates who will go on to make important contributions to society. And when it comes to driving innovation, governments in the developing world, with their limited resources, are expected to invest mostly in applied research, with the scientific agenda set primarily by the quest to find solutions to the practical problems facing their populations.

It is obviously important that African countries prioritize education and support applied research. However, I argue that it would be a grave mistake to do that in the absence of an excellent fundamental research agenda. Unless African countries aim for the highest levels of scientific research excellence within a milieu of unfettered inquiry, the continent will continue to languish on the treadmill of poverty and inequality. By striving for open-ended, curiosity-driven research, we will be better placed to harness the benefits of education and to stimulate innovation. While being attentive to our problems on the ground, we must aim for the sky. 
Astronomy in South Africa offers an enlightening example. The construction of the MeerKAT radio telescope-named after a beloved mammal from the Karoo-cost the South African government more than 5 billion South African rand $(\$ 334$ million). A precursor to an even more ambitious project-the Square Kilometre Array-MeerKAT is the most sensitive telescope in a radio-frequency range that is important for cosmology and astronomy. Radio astronomy may appear to be far from everyday life-how does the the study of distant planets, stars, and galaxies put a roof over one's head or a plate of food on the table? But we must keep in mind that an important part of research is to attract new generations into science.

In my view, astronomy is arguably the discipline that can best achieve this goal across cultures, geographical regions, and generations. Crucially, this field can create hope, whose importance in Africa can't be underestimated. MeerKAT has energized science teachers, engaged the public, and educated hundreds of graduate students who have found employment either at home or in another African country. What is more, astronomy has exposed our best students to new cutting-edge problems in computing, communication, data science, mechanics, electronics, and optics, which all are applicable to other fields.

The response of researchers to the pandemic is one compelling demonstration of the tangible benefits of fundamental research for the real world. When COVID-19 hit South Africa, particle physicists at the University of the Witwatersrand (Wits) applied artificial intelligence and machine-learning methods to monitor and predict the evolution of the pandemic, providing valuable input to government policies that helped make life-saving decisions. Building on their successes in South Africa, those researchers are now helping other African countries.

So what can developing countries learn from this success? How can they tap the applied potential of fundamental research without diluting the rigor of academic degrees and without turning universities into technology centers that merely support industry? A promising approach is to expose students to the ideas of innovation during the course of their graduate degrees rather than after them. At Wits, we are piloting a dual degree program where selected doctoral students from different disciplines simultaneously complete an M.Sc. in innovation. While pursuing their Ph.D.'s, the students learn about entrepreneurship and work with mentors who transitioned ideas from fundamental inquiry to commercial success.

We are confident the program will demonstrate that fundamental inquiry and innovation aren't mutually exclusive. If we educate bright, inspired students, it won't matter whether they turn their attention to string theory or commercial devices-they will simply change our societies for the better.

Nithaya Chetty: University of the Witwatersrand, Johannesburg, South Africa 